\title{
Gold-Silver Ratio and Its Utilisation in Long Term Silver Investing
}

\author{
Ing. Peter Arendas, PhD
}

\author{
Department of Banking and International Finance, Faculty of National Economy \\ University of Economics in Bratislava; Email: p.arendas@centrum.sk
}

\section{Doi:10.5901/mjss.2016.v7n1p285}

\begin{abstract}
Gold-silver ratio is one of the best known ratios of commodity markets. The historical gold-silver ratio used to be approximately 15 which is almost in line with the estimates of geologists that there is 19 times more silver than gold in the Earth's crust. The gold-silver ratio used to be administratively set during the era of bimetallism. But after silver and later also gold were eliminated from the monetary system, the gold-silver ratio started to fluctuate wildly. The gold-silver ratio is closely followed by many financial analysts and investors who try to estimate the future price development of both of the metals. The aim of this article is to find out whether the gold-silver ratio may be used as a cornerstone for a profitable long term investment strategy. The research shows that when investing in silver, a simple strategy based on the gold-silver ratio brings higher returns than the buy \& hold strategy. The difference between returns of the two strategies is statistically significant in the long run.
\end{abstract}

Keywords: gold, silver, investing, gold-silver ratio, buy \& hold

\section{Introduction}

Gold-silver ratio is one of the most closely followed ratios of the commodity markets. Gold as well as silver are precious metals and their prices are impacted by factors such as inflation expectations or fear. On the other hand silver has much wider industrial usage which means that silver price is significantly affected also by the industrial demand. As a result, both of the metals have different dynamics of price development. Although they tend to move in the same direction, the pace of price growths and declines is different. Silver price is more volatile and its price moves tend to be stronger. As a result, the gold-silver ratio isn't stable but it changes remarkably over time. The aim of this article is to find out whether the gold-silver ratio may be used as a cornerstone for a profitable long term investment strategy.

There are several authors that focus their attention on development of precious metals prices, on relations between particular precious metals or on precious metals investment strategies. For example Senoy (2013) studied the volatility shifts in returns of gold, silver platinum and palladium from 1993 to 2013. He concluded that the financial crisis of 2008 led to increased volatility of platinum and palladium prices, however no significant impact on volatility of gold and silver prices was observed. He also concluded that precious metals prices get strongly correlated after 2000.

Bagautdinova, Tsaregorodtsev, Kulalayeva and Arzhantseva (2014) analysed the ability of gold, silver, platinum and palladium to protect money from exchange rate depreciation from the point of view of a Russian investor. They concluded that silver, platinum and palladium are more suitable for hedging purposes due to their wider industrial usage and resulting higher industrial demand.

On the other hand Bunnag (2015) who analysed precious metals volatility co-movements and spill-overs tried to find an optimal two-metal portfolio. He came to conclusion that an optimal portfolio should contain more gold than other precious metals in order to minimise risk without lowering the expected return.

Elder, Miao and Ramchander (2012) analysed the impact of US macroeconomic news on return, volatility and trading volume of gold, silver and copper futures. They found out that the reactions on surprising economic news are swift and significant and that the news about an unexpected improvement in the economy have negative impact on gold and silver prices and positive impact on copper prices.

An interesting study was undertaken by Lucey and Li (2015) who concluded that not only gold but also silver, platinum and palladium may act as a safe haven and that there are time periods when silver, platinum and palladium act as safe haven while gold doesn't.

Mutafoglu, Tokat and Tokat (2012) analysed whether trader positions can predict the direction of gold, silver and platinum prices. They concluded that there is a positive correlation between returns and net positions of non-commercial and non-reporting traders and that extreme trader positions have some ability to forecast market returns.

A similar research was conducted by Bosch and Pradkhan (2015) who analysed the impact of speculators on 
precious metals futures markets. They found out that non-commercial trader's positions had a destabilizing impact on returns and volatility of precious metals futures prices before June 2006, although they couldn't confirm the same effect after June 2006.

Narayan, Narayan and Sharma (2013) studied the relations between commodity futures markets and commodity spot markets and the ability of the futures market to predict spot prices. The study involved gold, silver, platinum and oil. They found out that the information provided by the futures markets can be used to create profitable trading strategies.

Hassani, Silva, Gupta and Segnon (2015) analysed the ability of 17 different forecasting methods to provide accurate and statistically significant forecasts of gold prices. They came to conclusion that none of the methods was able to outperform the random walk at horizons of one and nine steps ahead. The exponential smoothing model was able to provide most accurate predictions on average.

The relationship between gold and silver prices was studied by Baur and Tran (2014). They analysed the relationship between gold and silver over the 1970-2011 time period and the impacts of bubbles and financial crises on this relationship. They concluded that there is a clear evidence of a co-integration relationship with gold prices driving the gold-silver relationship. They also concluded that bubbles and financial crises impact the relationship.

Paschke and Prokopczuk (2012) analysed the ability of continuous time pricing models to identify mispriced commodity futures contracts. They analysed gold, silver, copper and oil markets. Their results showed that the models are able to identify mispriced contracts and that investment strategies based on the pricing errors are able to generate excess returns.

As the short literature review shows, the researchers focus on various aspects of the precious metals markets, however only little attention is paid to the gold-silver ratio and its applicability in the investment process.

\section{Data and Methodology}

The aim of this article is to find out whether gold-silver ratio is suitable as a basis for profitable long term investment strategies. There are three main mechanisms that lead to a decrease or an increase of the gold-silver ratio. The ratio grows if gold price grows while silver price declines, or if gold price grows quicker than silver price or if it declines slower than silver price. And vice versa, gold-silver ratio declines if silver price grows while gold price declines, or if silver price grows quicker than gold price or if silver declines slower than gold price. Silver prices are more volatile compared to gold prices. This is why silver prices mostly grow quicker and also fall quicker than gold prices. It means that bull precious metals markets are usually characterised by decreasing gold-silver ratio and bear precious metals markets are usually characterised by increasing gold-silver ratio.

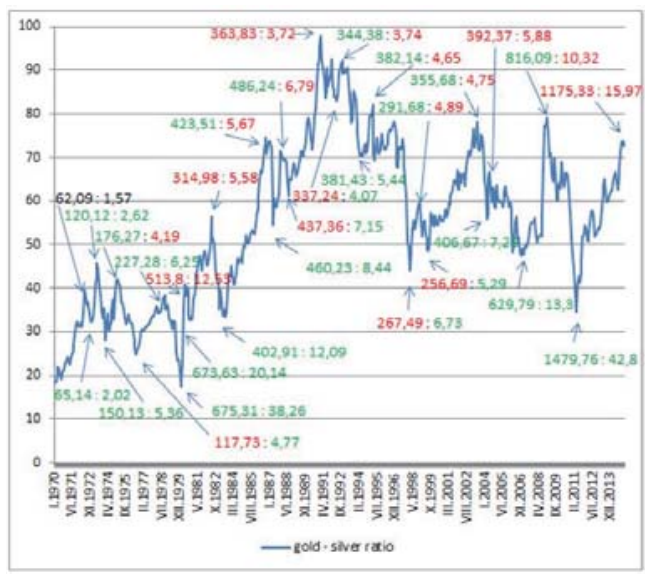

Figure 1: Historical development of gold-silver ratio (1970 - 2015)

Source: own processing, using data of the IMF

Figure 1 shows development of the gold-silver ratio over the last 45 years. The ratio is based on average monthly prices reported by statistical databases of the IMF. The chart shows the gold-silver ratio as well as the gold and silver prices 
related to every major top and bottom. It is able to see that most of the tops occurred after gold price increased compared to the previous bottom. But what is more important, in all of the cases, the bottoms occurred after silver price increased compared to the previous top. It means that gold-silver ratio is more efficient in identifying silver growth trends than in identifying gold growth trends. This is why this paper is focused on silver investing rather than on gold investing.

Two hypotheses will be tested:

- $\quad \mathrm{H} 1$ : It is able to create a long term investment strategy that will be focused on silver investing, using the goldsilver ratio to generate buy and sell signals and that will be more profitable than a simple buy \& hold strategy in the long term.

- H2: The difference between the returns of the gold-silver ratio based investment strategy (GSR strategy) and buy \& hold strategy are statistically significant.

The silver investing strategy analysed in this paper is based on buying silver when the gold-silver ratio approaches its local peak and selling it when the gold-silver ratio approaches its local bottom. However it is easy to identify the peaks and bottoms ex-post, it is extremely hard to identify it ex-ante. Moreover using the gold-silver ratio on a daily basis may lead to a higher level of false signals. This is why there are a couple of assumptions related to the strategy:

1. Monthly gold and silver prices are used to calculate the gold-silver ratio. The gold-silver ratio is calculated as follows:

$$
G S R_{n}=\frac{A u_{n}}{A g_{n}}
$$

$\mathrm{GSR}_{\mathrm{n}}-$ Gold-silver ratio in time $\mathrm{n}$

Aun - Gold price in time n

$\mathrm{Ag}_{\mathrm{n}}$ - Silver price in time $\mathrm{n}$

2. Bollinger Bands are used to generate buy and sell signals. The Bollinger Bands are calculated as follows:

$U B B=G S R M A_{12}+2 * G S R S T D_{12}$

$L B B=G S R M A_{12}-2 * G S R S T D_{12}$

UBB - Upper Bollinger Band

LBB - Lower Bollinger Band

GSRMA $_{12}$ - Gold-Silver Ratio Moving Average for 12 periods

GSRSTD 12 - Gold-Silver Ratio Standard Deviation for 12 periods

3. Although it is easy to go short silver using the modern financial tools such as ETFs, it used to be a much more complicated process in the past and derivative contracts were needed. Given that financial derivatives bring additional risks and higher additional costs, this study is based on investing in physical silver that is bought or sold at the recent LBMA silver fix price. As a result, only long positions are initiated, the sell signals serve only to liquidate the long positions.

4. If the gold-silver ratio crosses the upper Bollinger band to the upside, a long position in silver is initiated on the first trading day of the following month, using the LBMA silver fix price for that day. If $G_{S R}$ is value of the goldsilver ratio calculated for month $n$ and $U_{B B}$ is value of the Upper Bollinger Band calculated for month $n$, then the buy signal is generated as follows:

- $\mathrm{GSR}_{n}>\mathrm{UBB}_{\mathrm{n}} \rightarrow$ a long position is initiated on the first trading day of month $\mathrm{n}+1$, using the LBMA silver fix price for that day

5. If the gold-silver ratio crosses the lower Bollinger band to the downside, the long position in silver will be liquidated on the first trading day of the following month, using the LBMA silver fix price for that day. If GSR $\mathrm{R}_{\mathrm{n}}$ is value of the gold-silver ratio calculated for month $n$ and $L_{B B}$ is value of the Lower Bollinger Band for month $n$, then the sell signal is generated as follows:

- $\mathrm{GSR}_{\mathrm{n}}<\mathrm{LBB}_{\mathrm{n}} \rightarrow$ any open position is liquidated on the first trading day of month $\mathrm{n}+1$, using the LBMA silver fix price for that day

6. If a buy signal is generated when a long position has been already opened, no action is taken. I.e. there is no more than one position opened at any particular time.

7. The abovementioned investment strategy is applied on 10-year, 20-year and 30-year time intervals.

8. Method of moving time intervals is used to get statistically more significant results. For example the first 10year interval covers years 1970-1979, the second one covers years 1971-1980, etc. As a result, the strategies were tested on 36 10-year intervals, 2620 -year intervals and 1630 -year intervals.

9. The buy \& hold strategy assumes that a long position is initiated on the first trading day of the time period, using the LBMA silver fix price and it is liquidated on the first trading day following the end of the time period, using the LBMA silver fix price. For example for the 10-year period 1970-1979, a long position is initiated on 
January 2, 1970, at silver price of $\$ 1,8 /$ troy ounce (toz) and it is liquidated on January 2, 1980, at silver price of $\$ 39,95 /$ toz.

10. The GSR strategy assumes that a long position is initiated after a buy signal was generated and it is liquidated after a sell signal was generated. There can be multiple trades realized during the time period, but there cannot be opened more than one position at a time. For example during the first 10-year time period, there are two trades made. Silver is bought on October 1,1971 , at $\$ 1,38 /$ toz and sold on July 1,1976 , at $\$ 4,82 /$ toz and it is bought again on September 1, 1978, at $\$ 5,519 /$ toz and sold on October 1, 1979, at $\$ 17,09 /$ toz. What is important, there is always the whole account balance invested into any trade. It means that the gains from a trade are fully reinvested into another trade. On the other hand if a trade generates a loss, the next trade is negatively impacted, as less money can be deployed. If there is a position opened at the end of the time period, it is liquidated on the first trading day following the end of the period.

11. Returns of the GSR strategy and buy \& hold strategy for every particular time interval are analysed using the Wilcoxon Signed-Ranks Test, to evaluate the statistical significance of the results.

\section{Results}

As Figure 2 shows, the longer the time period, the more superior is the GSR strategy. The buy \& hold strategy is better only for 10-year time periods. 36 10-year time periods were analysed and the average return of the buy \& hold strategy was $186,41 \%$. However, the GSR strategy lagged only slightly, as it was able to generate average return of $173,25 \%$. A huge advantage of the buy \& hold strategy is that it is able to capture the whole extent of major bull runs, while the GSR strategy is able to capture only the middle part of the bull runs. On the other hand the buy \& hold strategy also captures the whole extent of bear runs, while the GSR strategy is able to avoid a meaningful part of them. The positive aspects of the GSR strategy need more time to accumulate and show the positive effects.

The analysis shows that the GSR strategy is far superior to the buy \& hold strategy during the 20-year time periods. There were 2620 -year periods analysed and the GSR strategy was able to generate returns of $258,69 \%$ on average, while the buy \& hold strategy reached an average return of only $138,39 \%$. The difference was even bigger when taking into account the 30 -year time period, where the score was $522,84 \%: 139,07 \%$ in favour of the GSR strategy.

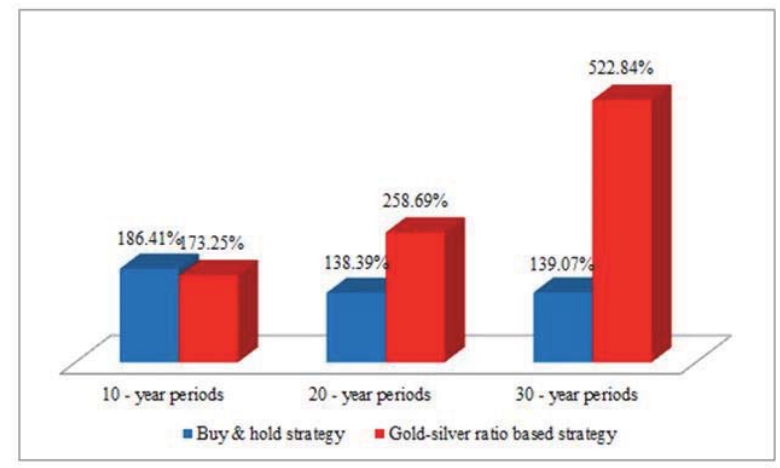

Figure 2: Average returns of the strategies for various time period groups

Source: own processing

The results of the strategies don't have a normal distribution. This is why the standard Paired-Sample t-test may generate inaccurate results. Therefore the statistical significance of the results is tested using the non-parametric Wilcoxon SignedRank test.

Figure 3 shows the results of the Wilcoxon Signed-Rank Test for the results of the 10-year periods. The difference between results of the buy \& hold strategy and the GSR strategy was only small. The Wilcoxon Signed-Rank Test provided two-tailed $p$-value of 0,555763 which means that the null hypothesis cannot be rejected. It indicates that the difference between the results of the buy \& hold strategy and the GSR strategy is not statistically significant. 
Figure 3: Wilcoxon Signed-Rank Test (10-year periods)

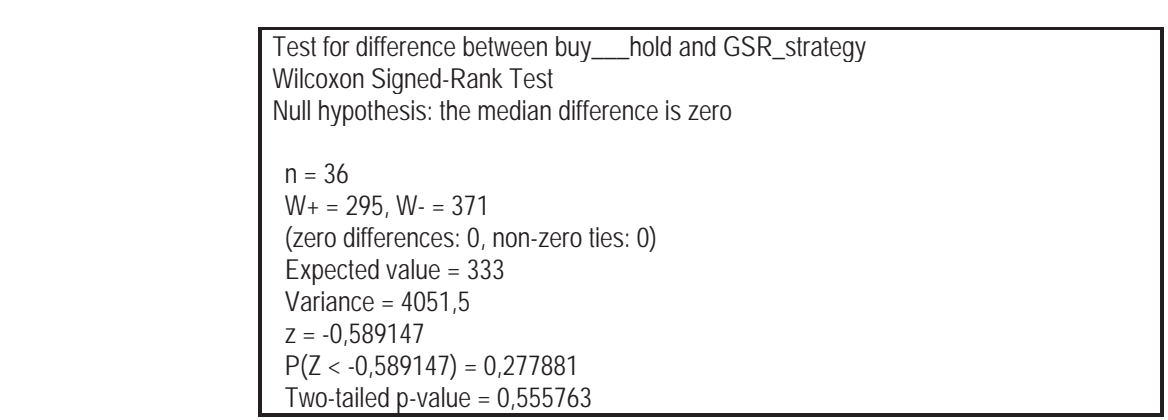

Source: own processing, using Gretl

Results of the Wilcoxon Signed-Rank Test for the 20-year periods are presented by Figure 4. Although the average returns were highly in favour of the GSR strategy, the Wilcoxon Signed-Rank Test provided two-tailed p-value of 0,162447 . Although the $p$-value is significantly lower compared to 10 -year periods, it is still higher than the critical value of 0,05 . It means that the null hypothesis cannot be rejected and the difference between the results of the buy \& hold strategy and the GSR strategy may be marked as not statistically significant.

Figure 4: Wilcoxon Signed-Rank Test (20-year periods)

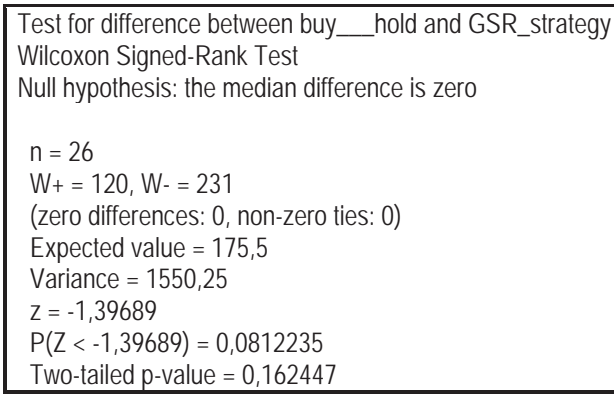

Source: own processing, using Gretl

In the case of the 30-year time periods, the difference between returns of the buy \& hold strategy and the GSR strategy was huge. It is confirmed also by the results of the Wilcoxon Signed-Rank test. The final $p$-value is 0,00102524 which means that the null hypothesis may be rejected. The rejection of the null hypothesis indicates statistical significance of the difference between results of the buy \& hold and the GSR strategy.

Figure 5: Wilcoxon Signed-Rank Test (30-year periods)

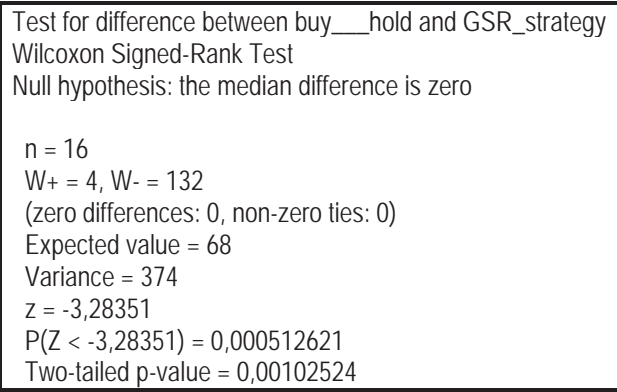

Source: own processing, using Gretl 


\section{Discussion}

The presented data show that it is possible to build a successful long term investment strategy using the gold-silver ratio to generate buy and sell signals. The comparison of the simple buy \& hold strategy and the GSR strategy shows an evident dominance of the GSR strategy during longer time periods. The analysis has shown that one of the hypotheses can be accepted and the second one cannot be rejected.

Hypothesis $\mathrm{H} 1$ (It is able to create a long term investment strategy that will be focused on silver investing, using the gold-silver ratio to generate buy and sell signals and that will be more profitable than a simple buy \& hold strategy in the long term) can be accepted. Although the buy \& hold strategy brought better results on the 10 -year time periods, the GSR strategy was dominant during the 20-year and 30-year time periods.

Hypothesis H2 (The difference between the returns of the GSR strategy and the buy \& hold strategy is statistically significant.) cannot be rejected. Although the analysis showed that the differences between average returns of the two strategies are not statistically significant regarding the 10-year and 20-year time periods, the difference is statistically significant regarding the 30 -year time periods.

\section{Conclusion}

The gold-silver ratio is an important indicator that can provide useful information about the current state of the precious metals markets. The empirical analysis shows that major increases of the gold-silver ratio are mostly related to growing gold prices and declining silver prices. The major declines of the gold-silver ratio are always related to growing silver prices. This is why this article focused on silver investing strategies, as the gold-silver ratio seems to be more reliable in predicting silver price movements than in predicting gold price movements.

The gold-silver ratio proved to be a highly reliable indicator of future silver price development. It is able to create investment strategies that use the gold-silver ratio as a generator of buy and sell signals. One simple silver investing strategy that uses the gold-silver ratio to generate buy and sell signals was presented in this paper. The results show that it is clearly superior to simple buy \& hold strategy in the long term. The differences between the average results of the two strategies are statistically significant regarding the long term (30-year) time intervals. Moreover there is a lot of room for optimisation of the strategy in order to achieve even better results.

\section{Acknowledgement}

This contribution is the result of the project implementation VEGA (1/0124/14) "The role of financial institutions and capital market in solving problems of the debt crisis in Europe".

\section{References}

Bagautdinova, N., Tsaregorodtsev, E., Kulalayeva, I., \& Arzhantseva, N. (2014). Assessment of mutual probabilistic influence of volatility of official price for precious metals on the market value of the Bi-currency basket. Mediterranean Journal of Social Sciences, 5 (12), 33-38.

Baur, D.G., Tran, \& D.T. (2014). The long-run relationship of gold and silver and the influence of bubbles and financial crises. Empirical economics, 47 (4), 1525-1541.

Bunnag, T. (2015). The precious metals volatility comovements and spillovers, hedging strategies in comex market. Journal of Applied Economic Sciences, 10 (1), 82-102.

Bosch, D., \& Pradkhan, E. (2015). The impact of speculation on precious metals futures markets. Resources Policy. 44, 118-134.

Elder, J., Miao, H., \& Ramchander, S. (2012). Impact of macroeconomic news on metal futures. Journal of Banking \& Finance, 36 (1), $51-65$.

Hassani, H., Silva, E.S., Gupta, R., \& Segnon, M.K. (2015). Forecasting the price of gold. Applied Economics, 47 (39), $4141-4152$.

Lucey, B.M., \& Li, S. (2015). What precious metals act as safe havens and when? Some US evidence. Applied Economics Letters, 22 (1), 35-45.

Mutafoglu, T.H., Tokat, E., \& Tokat, H.A. (2012). Forecasting precious metal price movements using trader positions. Resources Policy, 37 (3), 273-280.

Narayan, P.K., Narayan, S., \& Sharma, S.S. (2013). An analysis of commodity markets: What gain for investors? Journal of Banking \& Finance, 37 (10), 3878-3889.

Paschke, R., Prokopczuk, \& M. (2012). Investing in commodity futures markets: can pricing models help? European Journal of Finance, 18 (1), 59-87.

Sensoy, A. (2013). Dynamic relationship between precious metals. Resources Policy, 38 (4), 504-511. 